\title{
Research on marketing strategy of Chinese enterprises based on Data Mining Technology
}

\author{
ZhangZhuoqing, WuHuajuan \\ Nanchang Institute of Science \& Technology, Nanchang 330108,China
}

Key words: database; Internet; artificial intelligence; data mining; marketing

\begin{abstract}
The information flow under the Internet involves all aspects of all walks of life, access to valuable information means the opportunity to develop. In the era of knowledge explosion, the rapid change of information has promoted the update speed of knowledge, the traditional forms of information transmission has been unable to meet the needs of today's social development, the emergence of data mining and Internet has laid the foundation for the rapid spread of information, which gradually influenced and changed the way people access to information and means. At present, under the background of big data, it is a new field for Chinese enterprises that the model of enterprise marketing strategy based on data mining, and with the development of Internet, database and artificial intelligence, it has been playing its superiority. However, the data mining is still in the development stage, the system of marketing strategy based on data mining is not perfect, there are still many problems to be solved.
\end{abstract}

\section{Introduction}

In recent years, market has became more competitive than before, it has gradually evolved into the wisdom and ability of competition, society is becoming more and more dependent on computers, it can provide a theoretical basis for enterprise decision making through the algorithm to search deep information rules from large amounts of data, which puts forward new challenges and new methods for enterprises marketing strategy. At present, many enterprises use information management, but it is still difficult to obtain valuable information from the massive data, so easy to fall into the "data rich, lack of knowledge, lack of ways, the direction is unknown" dilemma in making the marketing strategy. To solve these problems above, enterprises need to strengthen the understanding of data mining and marketing database, and new technologies should be developed and the enterprise marketing strategy should be adjusted according to the actual situation of enterprises.

\section{Data mining technology}

Data mining is a kind of discovery of implicit, unknown, credible, novel and useful information for large data, it is an important field of database research. Therefore, data mining has obvious supporting role in determining the event strategy, the technology involves a wide field, and it is a cross subject, has been supported by statistics, machine learning, artificial intelligence, pattern recognition, knowledge database and visualization of multiple common foundation, and extended the function of statistical data formulation of business leaders, which has certain practical significance to adjust marketing strategy, and avoid risks. The relationship between data mining and a variety of disciplines is shown in Figure 1. 


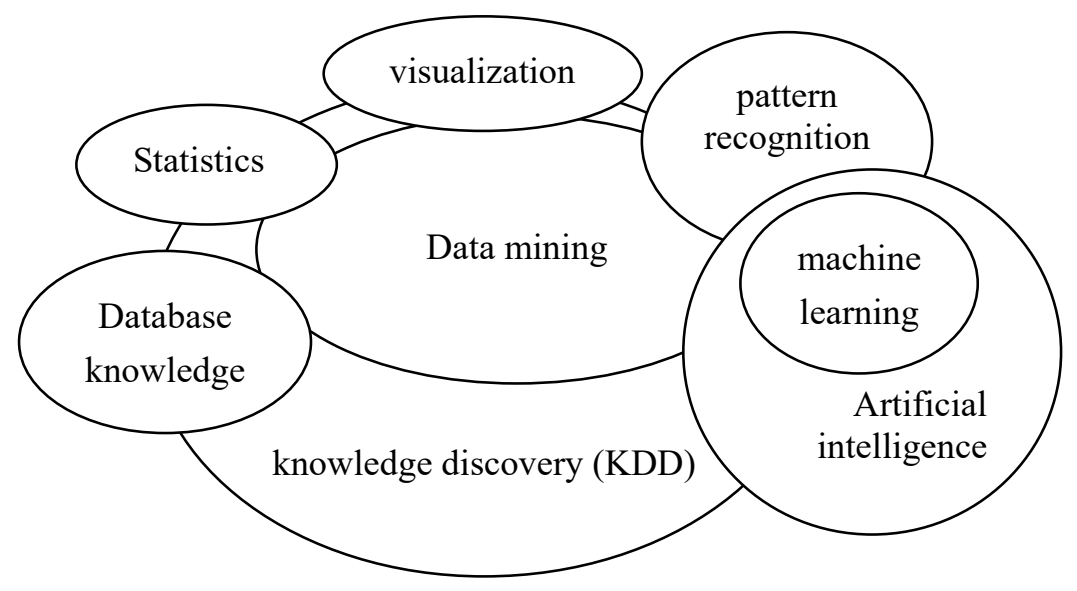

Figure 1 The relationship of data mining and a variety of disciplines

In daily life, people often confuse the data mining and knowledge discovery. In fact, knowledge discovery is a process that extracts potential valuable and can be processed directly from the database. "Model" is considered to be a test, improved knowledge. Knowledge discovery is a process to identify a certain pattern representation from big data, the process is a process of multiple steps, constantly adjust and spiral, the simple point that is found on the useful knowledge from a stack of data. Knowledge discovery includes 6 steps: cleaning, transformation, integration, mining, evaluation and performance, and data mining is the core of knowledge discovery. The flow chart of knowledge discovery is shown in figure 2.

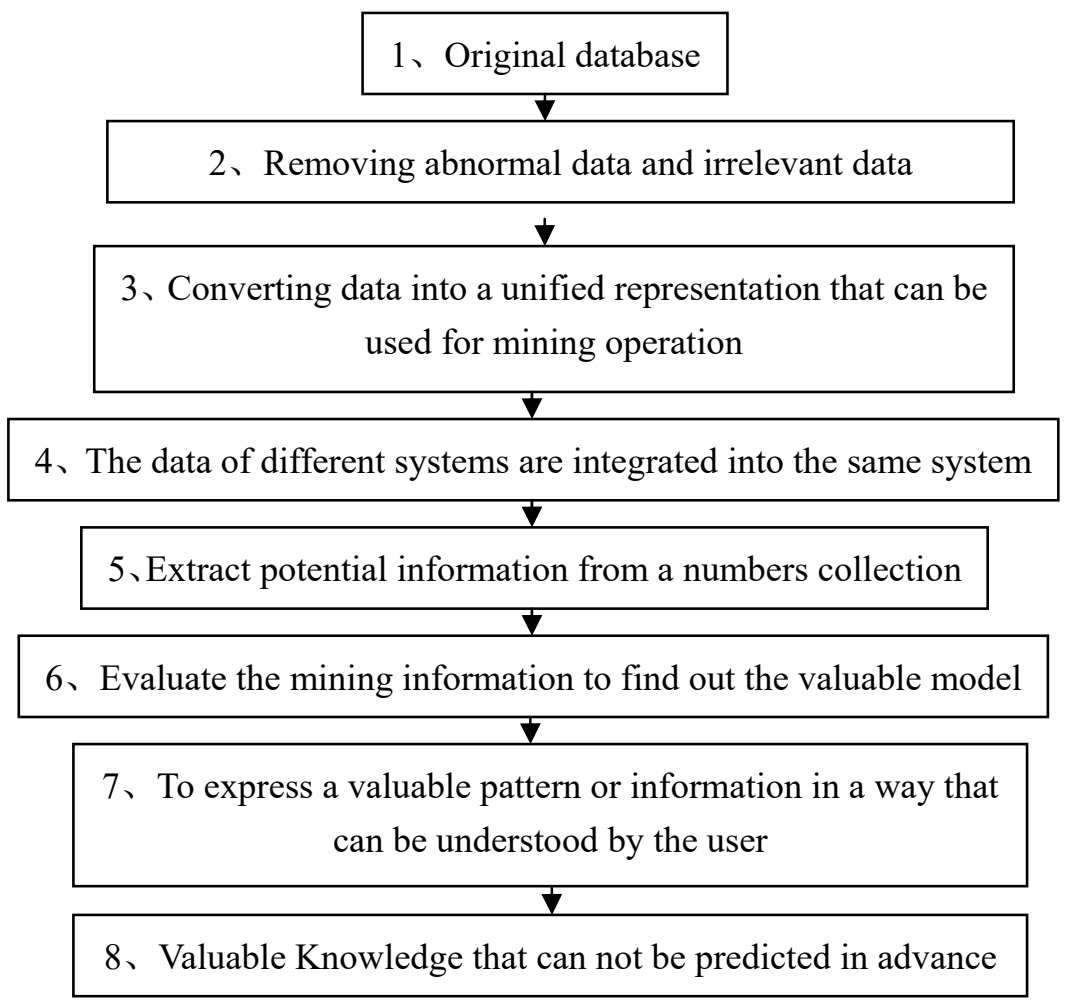

Fig. 2 The flow chart of knowledge discovery

At present, there are many analysis methods of data mining, which often need to be adjusted according to the practical problems. There are five methods of data mining, such as association analysis, sequence analysis, classification, prediction analysis and cluster analysis. 


\section{The application of data mining technology in database marketing strategy}

Data mining technology is used to find the potential knowledge in the big data, and then use the knowledge to solve the problem of "data rich and knowledge poor". With the rapid development of database and Internet technology, the application of data mining in database marketing strategy is more and more:

1. Customer analysis: through data mining analysis to understand customer information, looking for key customers, potential target customers and potential loss of customers, etc..

(1) association analysis. Analyze the purchasing habits and preferences of the customer groups to help identify the product positioning and related marketing strategies;

(2) cluster analysis. The customer groups to specific characteristics of the model segmentation, and then for various groups to take a unique marketing strategy;

(3) classification analysis. Establish the forecast model, simulate the response of the customer to the marketing strategy, and then optimize and improve according to the reaction, and realize the maximization of the profit.

2. Product analysis: to analyze the distribution and trend of sales of various products, determine the hot areas of various products; Determination of different seasons and regions, customer demand for the product level; and through the brand product sales from the market began to change, the life cycle, provide a strong basis for the timely adjustment of marketing strategy.

In addition, data mining is also applicable to the establishment of market forecasts, the development of enterprise resource planning, product pricing strategy, asset evaluation, cash flow analysis, etc..

\section{Enterprise marketing strategy model based on Data Mining}

For the marketing strategy model, domestic and international marketing experts and scholars have done a lot of research, a lot of well-known enterprises in the world have used this model to provide a scientific basis for marketing strategies, which can improve its accuracy.

In marketing, to carry out strategic decision process using data mining, the process is actually a large data centric, cycle and a number of factors closely linked, this process includes four business process, as shown in Figure 3:

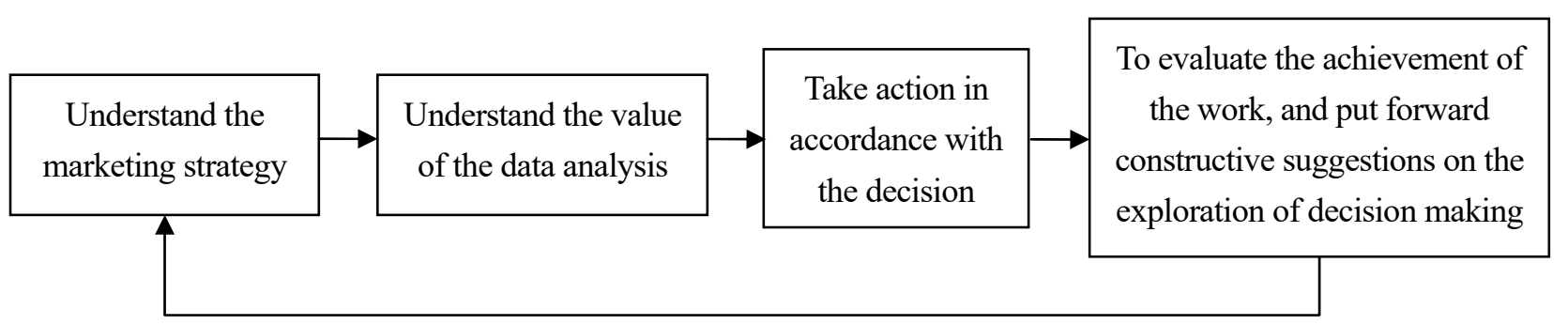

Fig. 3 Interactive cycle of enterprise marketing strategy based on Data Mining

For the interactive loop model, experts have conducted a lot of research, the most commonly used, and has become the industry standard is CRISP-DM cross industry data mining standards. The standard is not only the data presentation, not only is the analysis of modeling, but from business needs, develop programs to improve the practice of systems engineering. CRISP-DM cross industry data mining standards a total of business understanding, data understanding, data preparation, model building, program evaluation and implementation of the program six links. 
According to the interactive cycle process and CRISP-DM model of enterprise marketing strategy, we can get the enterprise marketing strategy model as shown in figure 4.

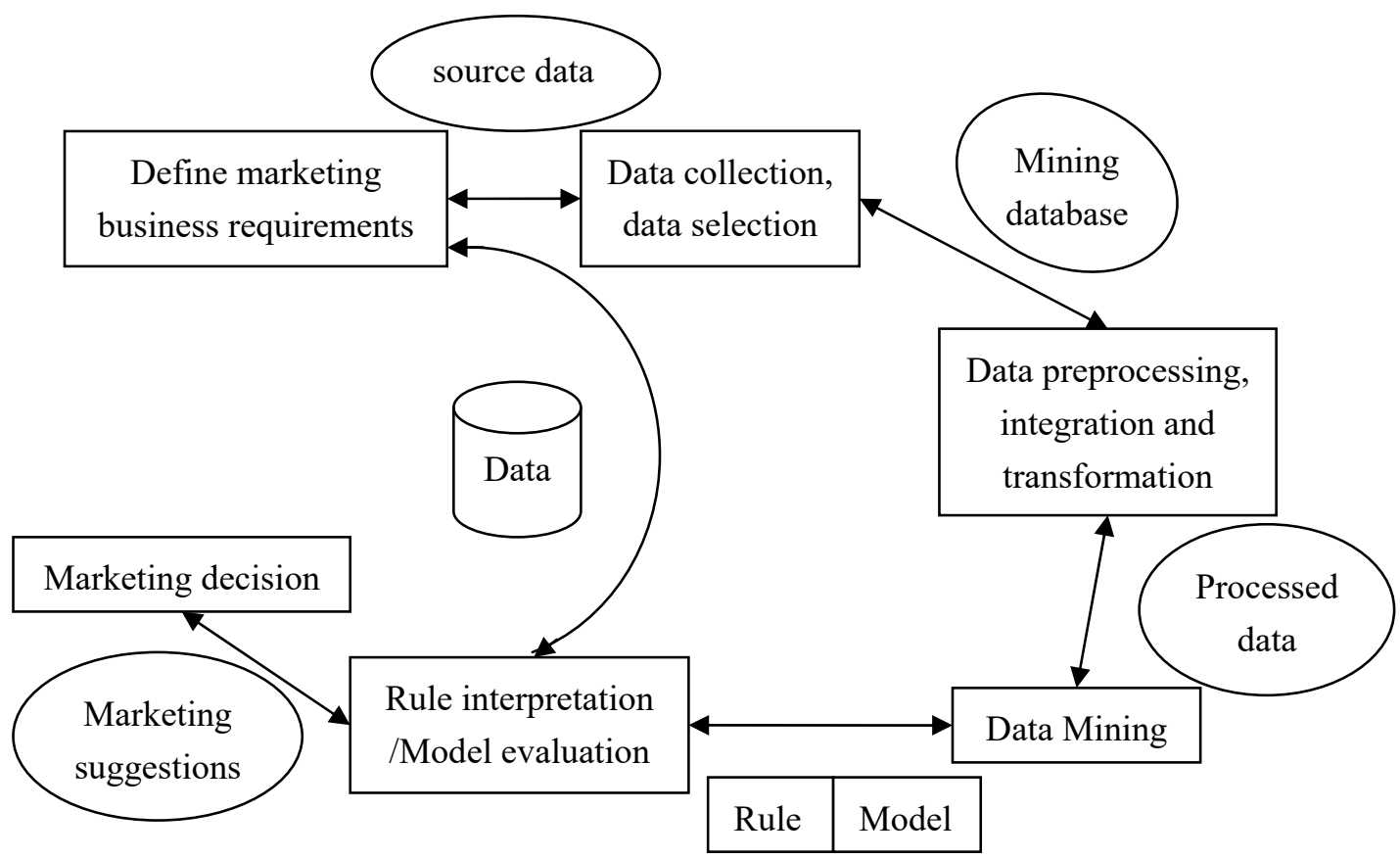

Figure 4 The enterprise marketing strategy model based on Data Mining Technology

We can see from Figure 4 that, the model mainly includes the marketing business needs and the definition of data collection, the establishment of marketing database, data preprocessing, data integration, data mining and decision analysis and interpretation of results, etc. The 7 part of the marketing strategy evaluation.

The data mining model is set up after a certain percentage of the original data out of test, the core algorithm of association rule mining is one of the Apriori algorithm, the Apriori algorithm has beeb used to establish a data mining model in this paper. Apriori algorithm flow is shown in figure 5.

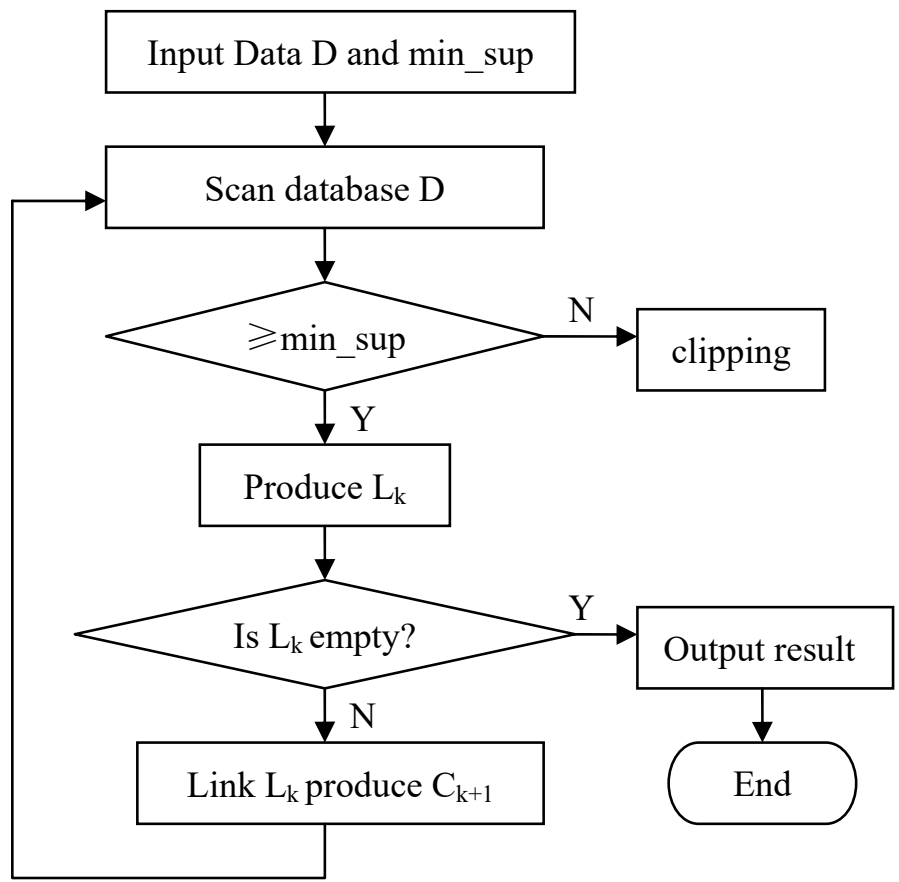

Fig. 5 The flow chart of Apriori algorithm 


\section{Conclusions}

Through the above discussion, the model of enterprise marketing strategy based on data mining is a new field of China's enterprises to explore, and with the development of Internet, database and artificial intelligence, it has been playing its superiority. In the knowledge economy era, marketing data mining can win the profit for the enterprise, but still need to expand the enterprise data search, reserve range, update large database and analysis method, more accurate and provide a theoretical basis for the enterprise market marketing strategy. In addition, it is needed for enterprises reserve data mining technology talent, and enhance the big data and artificial intelligence analysis, processing ability, and perfect combine data mining and marketing strategy, and explore the law of value persistence in the instantaneity of data information, which provide more comprehensive reference for enterprises.

\section{References}

[1] Hou Jin, Chen Cuicui. Research on the impact of marketing data mining on enterprise marketing strategy[J]. China Market,17, pp.39-40, 2014.

[2] Li Wenying. Telecom Customer Segmentation Based on data mining[D]. Xi'an University of technology, 2009

[3] Zhao Aiqin. Research and application of data mining in precision marketing in telecom industry[D]. Southwestern University of Finance and Economics, 2009

[4] Zhao Yun. Research on enterprise blog marketing strategy based on data mining[D]. Nanchang University, 2010.

[5] Li Wenguang. Business analysis system based on data mining technology[D]. Shandong University, 2005

[6] Fan Yi. Data mining based on telecom enterprise precision marketing[D]. Beijing University of Posts and Telecommunications, 2006

[7] Suo Qi. Database marketing research based on data mining[J]. Technology and Market, 2007,02, : 53-55.

[8] Chen Kai. Application of data mining in telecom industry CRM[D]. Jiangxi Normal University, 2013

[9] Long Man. Research on Customer Churn Management in telecom industry based on Data Mining[D]. Southwestern University of Finance and Economics, 2013

[10] Zhou Zhengwen, Zhang Jin. Research and application of data mining technology in 3G business expansion[J]. China Management Informationization, 2010,24: 49-52.

[11] Deng Xiaomei. Research on telecom customer segmentation model based on data mining[D]. Dalian University of Technology, 2006

[12] Li Jie. Telecom business analysis system - Design and implementation of data mart subsystem[D]. University of Electronic Science and technology of China, 2007

[13]Pang Qiaohong. The application of data mining technology in the analysis of credit card customer[D]. Southwestern University of Finance and Economics, 2012 